\title{
Light Source Halos in Night Vision Goggles: Psychophysical Assessments
}

\author{
Greg Craig $^{* a}$, Todd Macuda ${ }^{\mathrm{a}}$, Paul Thomas ${ }^{\mathrm{b}}$, Rob Allison $^{\mathrm{c}} \&$ Sion Jennings $^{\mathrm{a}}$ \\ ${ }^{a}$ National Research Council \\ Ottawa, Canada \\ ${ }^{\mathrm{b}}$ Topaz Technology Inc \\ Toronto, Canada \\ ${ }^{\mathrm{c}}$ York University \\ Toronto, Canada
}

\begin{abstract}
Anecdotal reports by pilots flying with Night Vision Goggles (NVGs) in urban environments suggest that halos produced by bright light sources impact flight performance. The current study developed a methodology to examine the impact of viewing distance on perceived halo size. This was a first step in characterizing the subtle phenomenon of halo. Observers provided absolute size estimates of halos generated by a red LED at several viewing distances. Physical measurements of these halos were also recorded. The results indicated that the perceived halo linear size decreased as viewing distance was decreased. Further, the data showed that halos subtended a constant visual angle on the goggles $\left(1^{\circ} 48^{\prime}, \pm 7^{\prime}\right)$ irrespective of distance up to $75^{\prime}$. This invariance with distance may impact pilot visual performance. For example, the counterintuitive apparent contraction of halo size with decreasing viewing distance may impact estimates of closure rates and of the spatial layout of light sources in the scene. Preliminary results suggest that halo is a dynamic phenomenon that requires further research to characterize the specific perceptual effects that it might have on pilot performance.
\end{abstract}

Keywords: Night vision goggles, lighting, halo.

\section{INTRODUCTION}

The safety of military night flying has increased greatly with the introduction of night vision goggles (NVGs). However, the visual input through NVGs differs greatly from the pilot's normal visual input in that it is monochromatic, the field of view is smaller, under poor lighting conditions the image can be noisy and the spectral sensitivity is different. Of the many visual artifacts that arise from NVGs, one that is of particular interest is the formation of halos. Halos result from viewing an NVG incompatible light source through NVGs as shown in the lower left hand side of Figure 1. In NVGs, halos are generated during the image intensification process and are seen as bright rings around light sources. Halos are produced by a variety of light sources and can be land, vehicle or aircraft based. Casual observation suggests that the perceptual attributes of halo (e.g. size, contrast, transparency) are influenced by the intensity, spectral composition and angular position of these light sources. In addition, overall ambient lighting conditions will also contribute to halo attributes. As a consequence, halo can be considered a complex perceptual phenomenon contributing to NVG produced imagery with possible counterintuitive visual characteristics. For example, anecdotally we have found that apparent halo size decreases as the observer moves closer to the light source. This is contrary to our every day visual experience and the laws of perspective where images of objects increase as the observer (or camera) moves closer. Human beings are sensitive to this relation and compensate for it in judging the apparent size of objects at different distances. Importantly these size constancy mechanisms in human vision are not easily overridden when viewing NVG images. Thus, it is not surprising that anecdotal reports from pilots indicate that they avoid directly viewing halos during flight as they tend to degrade NVG visuals. While there are an abundance of anecdotal reports on the impact of halo on flight visual performance, the available data published in the literature is lacking. Taken together these observations suggest that halo requires further study as it may interfere with precise aircraft control, target identification and recognition.

* greg.craig@nrc-cnrc.gc.ca; phone 1613 991-1997; fax 1613 952-1704

5800-7 V. 3 (p.1 of 5) / Color: No / Format: Letter / Date: 2005-04-12 16:22:56

SPIE USE: DB Check, Prod Check, Notes: 


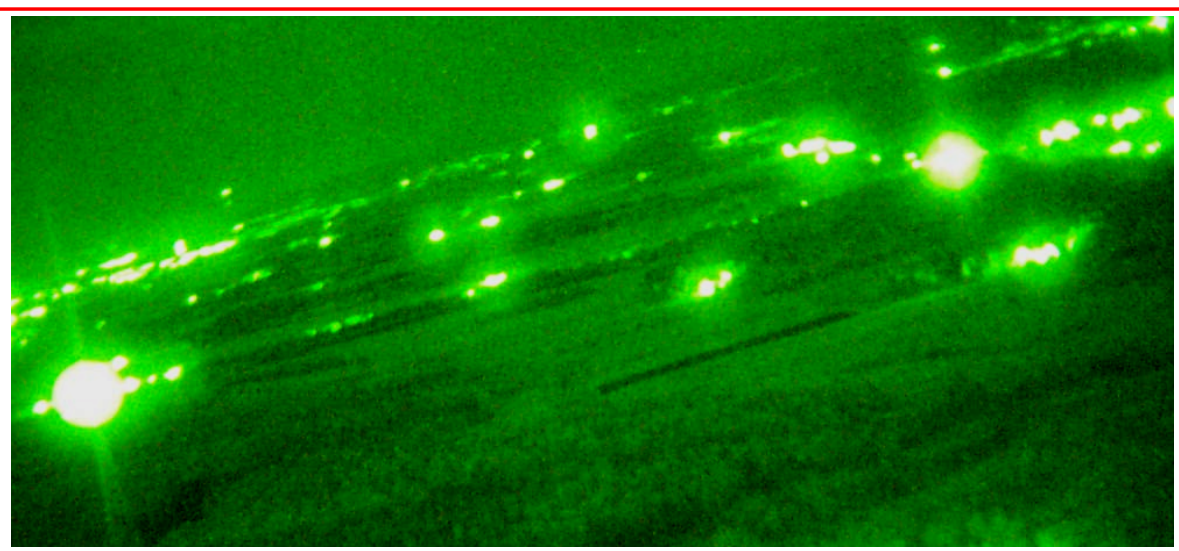

Figure 1: NVG incompatible lights and resultant halos

The effects of halo on perception and flight performance have not been well documented. To address this issue the current paper will focus on the perceptual characterization of halo. This was accomplished by developing a methodological paradigm that utilizes physical and psychophysical procedures to examine halo characteristics. This general purpose experiment was conducted to establish an initial methodology and technique to measure the perceptual attributes of halo. We present some initial data that provide insight into the halo phenomenon and illustrate the utility of the methodology.

\section{METHODS}

\subsection{Perceptual Characterization of halo: Methodological Development}

At present there is no well developed methodology for assessing the impact of halo on visual perception. The following study is a first step in this regard and can be considered a general purpose experimental paradigm for examining perceived angular size of halo at a series of viewing distances. This methodology was used to assess the counterintuitive observation of decreasing halo size as the observer moves closer to the light source. This observation is not consistent with normal visual mechanisms and is likely linked to the device characteristics of NVGs.

\subsection{Apparatus}

A set of Gen III, Omni 4 tubes were used to view the light source, which was a high brightness, single-color red LED (Stanley Electric FR5365S series ${ }^{1}$ ) with a dominant wavelength of $626 \mathrm{~nm}$, positioned centrally behind a target shown in Figure 2a. The target consisted of a series of concentric rings that had a line width of one inch and were separated by two inches. In general, the size and spacing of these rings could be modified to be appropriate for arbitrary target distance and precision requirements but the values used were a good compromise for the range of distances studied. The central area of the target was six inches in diameter. A Kodak DC4800 digital camera was focused on the NVG display to photograph the halos at the various distances tested. A small light was used to illuminate the target so that both the halo and the rings on the target were visible. 


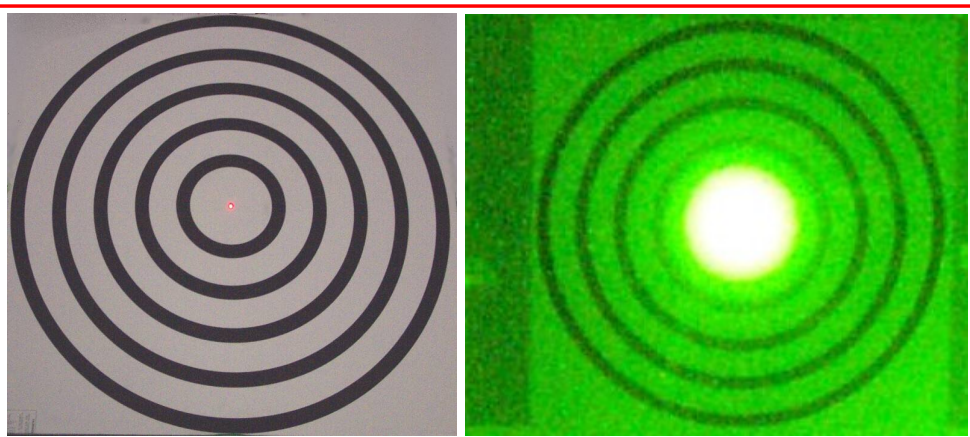

Figure 2: a) Target stimulus and central red LED and b) Target stimulus through NVG with small central halo

\subsection{Procedure}

The NVGs were mounted on a tripod that was placed 75, 50, 25 and 15 feet from the target and focused as required for each viewing distance. For each condition, two measurements of the halo size were conducted. For the psychophysical measurement, the subject viewed the halo and indicated how many rings of the target were obscured by the halo. Although the halo was composed of an intense area in the center and a less intense secondary surround, only the brightest, central region of the halo was measured. For example, in Figure 2b, the halo can be seen to cover the center and first ring of the target. This perceptual estimate of halo diameter was then converted to a perceived angular halo size.

For the physical measurement, a photograph was taken at each distance through the NVGs. Using this photograph, the size of the halo was measured with respect to the overall size of the field of view, which is known to be 40 deg (Figure 3). Figure 3 illustrates how CorelDraw Version 11 was used to determine the diameter of the full field of view (red ticks) and then calculate the diameter of the photographed halo (black ticks). The angular size of the halo was calculated as a proportion of the total field of view and then converted to linear size in inches.

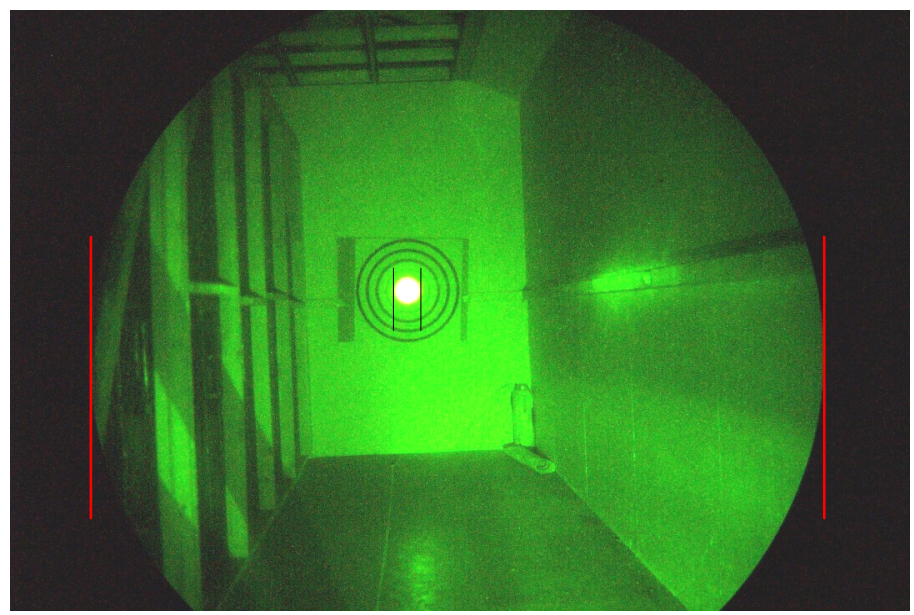

Figure 3: Halo picture capturing NVG full field of view

\section{RESULTS AND DISCUSSION}

\subsection{Perceived/Physical halo Size}

The results were similar for the physical and psychophysical measurements of the halo size, as can be seen in Table 1 . As distance from the target increased, the area subtended by the halo appeared to increase, covering more of the target. This effect was apparent both to the viewer and in the photographs of the stimulus (see Figure 4). Further analysis of the data indicated that the perceived visual angle of the halo remained relatively constant for all distances while the visual 
angle of the target increased as distance decreased. Thus, the constant halo visual angle, coupled with the increasing target visual angle caused the halo to appear to shrink as the viewer approached the light source.

\begin{tabular}{|c|c|c|c|c|}
\hline Distance & $\begin{array}{c}\text { Perceived } \\
\text { Halo Size }\end{array}$ & $\begin{array}{c}\text { Measured } \\
\text { Halo Size }\end{array}$ & $\begin{array}{c}\text { Perceived Halo } \\
\text { Visual Angle }\end{array}$ & $\begin{array}{c}\text { Measured Halo } \\
\text { Visual Angle }\end{array}$ \\
\hline $15^{\prime}$ & $6 ”$ & $5.52^{\prime}$ & $1.9^{\circ} \pm 0.2^{\circ}$ & $1.8^{\circ} \pm 0.2^{\circ}$ \\
\hline $25^{\prime}$ & $10^{\prime}$ & $10.44^{\prime}$ & $1.9^{\circ} \pm 0.2^{\circ}$ & $1.9^{\circ} \pm 0.2^{\circ}$ \\
\hline $50^{\prime}$ & $20^{\prime}$ & $18.72^{\prime}$ & $1.9^{\circ} \pm 0.2^{\circ}$ & $1.8^{\circ} \pm 0.2^{\circ}$ \\
\hline $75^{\prime}$ & $30^{\prime}$ & $26.52^{\prime}$ & $1.9^{\circ} \pm 0.2^{\circ}$ & $1.7^{\circ} \pm 0.2^{\circ}$ \\
\hline
\end{tabular}

Table 1: Psychophysical and measured halo size

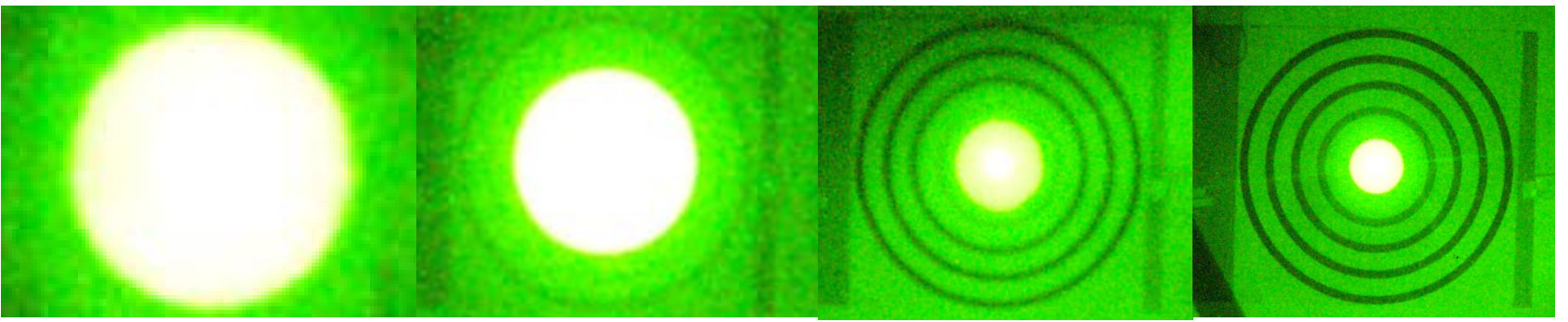

Figure 4: Halo size at 75, 50, 25 and 15 feet, from left to right.

\subsection{Expected Visual Angle of a 6" Target: Comparison to Halo size}

For comparison, we have plotted the expected visual angle for the image of a real 6" target (the size of the apparent halo at 15') at the various distances. It is clear that the halo image does not behave like the perspective projection of real objects when distance varies and is better fit by a constant angular size model. Since, the measured and perceived angular size of the halo remains constant an illusory reduction in size occurs as the observer moves closer to the light source (see Figure 4).

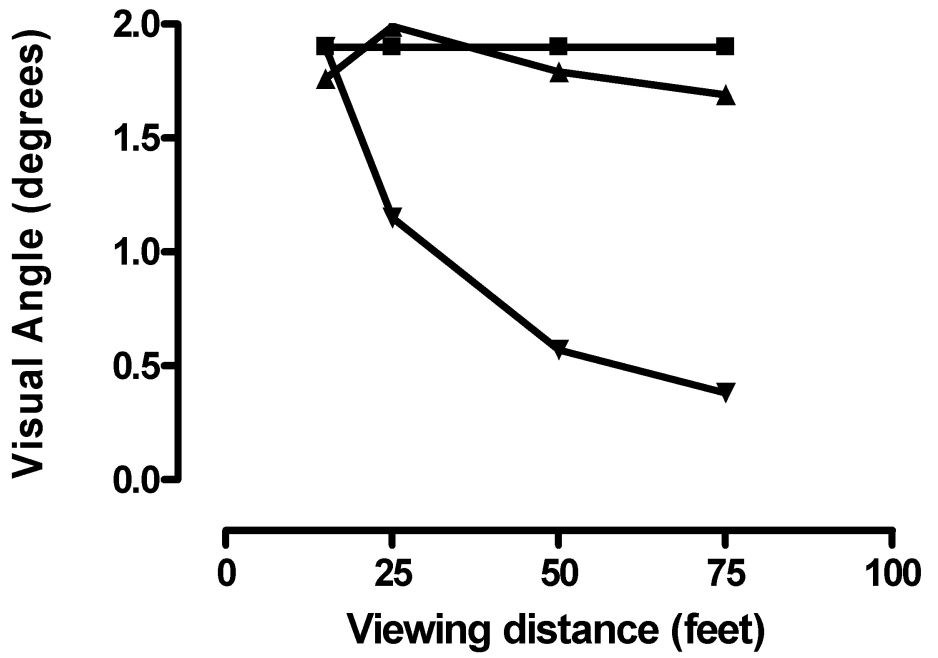

Figure 5: Perceived and Measured Angular Size versus Expected Angular Size 


\subsection{Implications for Flight}

The result of this experiment indicated that perceived halo size does not change with viewing distance. As shown above perceived and measured halo size is constant across viewing distance within a range of 75 '. Therefore, the apparent reduction in halo size with decreased viewing distance is an illusory effect. As a consequence, halo size is not an accurate cue for judging distance to a visual target. Under normal visual conditions the increase in visual angle of an object as it approaches the observer is a key element of estimating closure rates and object collision. ${ }^{2}$ It follows that pilots flying under NVGs could have difficulty estimating closure rates to objects/locations where halos are in the pilot's field of view. For example, as the pilot approaches a runway with NVG-incompatible light sources, the halos from the lights on the runway will apparently get smaller as the runway gets closer because the visual angle of the runway will increase, while the perceived visual angle of the halo remains constant. Under such conditions, use of halo cues would misinform the pilot that they were further away from the runway, producing a variety of potential errors in estimating the closure rate and time to contact with the runway. A potential outcome could be controlled flight into terrain.

Further, this effect could also apply to aircraft-based lights. Pilots could misjudge the distance to adjacent aircraft in a similar fashion. The results of the current study confirm that halo should be ignored during flight operations. However, the salience of these stimuli may make this task difficult.

\subsection{Relationship to Device Characteristics: Future Directions}

The constant perceived and measured size of halo suggests that this perceptual phenomenon is linked to the electrooptical characteristics of NVGs. Our research group recently developed a physical model of halo in NVGs (see Thomas et al., 2005, this issue). Our future efforts will combine the methodology developed in this study with more rigorous measurements of physical halo size. The thrust of these future research efforts will attempt to ameliorate the illusory halo size change described above with its constant perceived visual angle. Once we have validated the current findings we will examine other characteristics of light sources (e.g. intensity, spectral composition, angular position) that contribute to the phenomenology of halo.

\section{CONCLUSIONS}

In summary, the results of the current study support anecdotal observation of halo. These results indicate that halo size is not a valid cue for judging approach rates within a $75^{\prime}$ range. Halo could impair flight performance and should be ignored during all flight operations. The methods developed herein will serve useful in providing nominal perceived size estimates of halo in NVGs. Future studies will further characterize the phenomenology of halo at similar distances and those greater than 75 .

\section{REFERENCES}

${ }^{1}$ www.stanley-electric.com

2 Schiff, W., "The perception of impending collision: A study of visually directed avoidant behavior", Psychological Monographs, 79, 604-, 1965. 